\title{
Article
}

\section{Quantitative multiphoton microscopy of murine urinary bladder morphology during in situ uniaxial loading}

Hornsby, Jack, Daly, Donna, Grundy, David, Cheng, Fangzhou, Robertson, Anne M, Watton, Paul N and Thompson, Mark S

Available at http://clok.uclan.ac.uk/20222/

Hornsby, Jack, Daly, Donna ORCID: 0000-0001-9026-8200, Grundy, David, Cheng, Fangzhou, Robertson, Anne M, Watton, Paul N and Thompson, Mark S (2017) Quantitative multiphoton microscopy of murine urinary bladder morphology during in situ uniaxial loading. Acta Biomaterialia, 64 . pp. 59-66. ISSN 1742-7061

It is advisable to refer to the publisher's version if you intend to cite from the work. http://dx.doi.org/10.1016/j.actbio.2017.09.029

For more information about UCLan's research in this area go to http://www.uclan.ac.uk/researchgroups/ and search for <name of research Group>.

For information about Research generally at UCLan please go to http://www.uclan.ac.uk/research/

All outputs in CLoK are protected by Intellectual Property Rights law, including Copyright law. Copyright, IPR and Moral Rights for the works on this site are retained by the individual authors and/or other copyright owners. Terms and conditions for use of this material are defined in the policies page.

\section{CLoK}

Central Lancashire online Knowledge www.clok.uclan.ac.uk

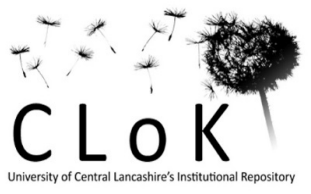




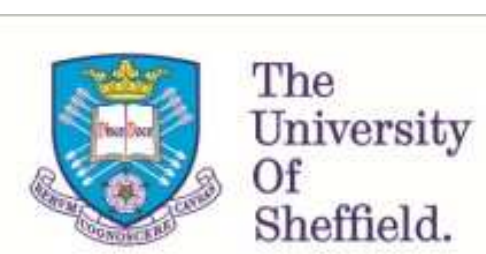

This is a repository copy of Quantitative multiphoton microscopy of murine urinary bladder morphology during in situ uniaxial loading.

White Rose Research Online URL for this paper:

http://eprints.whiterose.ac.uk/122933/

Version: Accepted Version

\section{Article:}

Hornsby, J., Daly, D., Grundy , D. et al. (4 more authors) (2017) Quantitative multiphoton microscopy of murine urinary bladder morphology during in situ uniaxial loading. Acta Biomaterialia. ISSN 1742-7061

https://doi.org/10.1016/j.actbio.2017.09.029

\section{Reuse}

This article is distributed under the terms of the Creative Commons Attribution-NonCommercial-NoDerivs (CC BY-NC-ND) licence. This licence only allows you to download this work and share it with others as long as you credit the authors, but you can't change the article in any way or use it commercially. More information and the full terms of the licence here: https://creativecommons.org/licenses/

\section{Takedown}

If you consider content in White Rose Research Online to be in breach of UK law, please notify us by emailing eprints@whiterose.ac.uk including the URL of the record and the reason for the withdrawal request. 


\section{Quantitative multiphoton microscopy of murine urinary bladder morphology during in situ uniaxial loading}

Jack Hornsby ${ }^{1}$, Donna M Daly ${ }^{2}$, David Grundy ${ }^{3}$, Fangzhou Cheng ${ }^{4}$, Anne M Robertson ${ }^{4}$, Paul N Watton ${ }^{4,5}$, Mark S Thompson* ${ }^{1}$

${ }^{1}$ Institute of Biomedical Engineering, University of Oxford, United Kingdom

${ }^{2}$ School of Pharmacy and Biomedical Sciences, University of Central Lancashire, United Kingdom

${ }^{3}$ Department of Biomedical Science, University of Sheffield, United Kingdom

${ }^{4}$ Mechanical Engineering and Materials Science, University of Pittsburgh, US

${ }^{5}$ Department of Computer Science \& INSIGNEO Institute for In Silico Medicine, University of Sheffield, United Kingdom

* E-mail: mark.thompson@eng.ox.ac.uk

Submitted to Acta Biomaterialia. Revised 18/9/17

\section{Keywords}

Multiphoton Fluorescence Microscopy, Bladder, Collagen, Mechanical Stress 


\section{Abstract}

Urodynamic tests are the gold standard for the diagnosis of bladder dysfunction, and the mechanical compliance of the bladder is an important parameter in these tests. The bladder wall has a layered structure, differentially affected by pathology, so knowledge of the contribution and role of these layers and their constituents to overall bladder compliance will enhance interpretation of these clinical tests. In this study we document the functional morphology of the detrusor and lamina propria of the murine bladder wall using a custom in-situ tensile loading system under multiphoton microscopy (MPM) observation in unloaded state and under incremental uniaxial stretch. Features in the stress-stretch curves of bladder samples were then directly related to corresponding MPM images. Collagen organisation across wall depth was quantified using image analysis techniques. The hypothesis that the lamina propria deformed at low strain by unfolding of the rugae and rearranging collagen fibrils was confirmed. A novel 'pocket' feature in the detrusor was observed along with extensive rearrangement of fibrils in two families at different depths, providing higher stiffness at high stretches in the detrusor. The very different deformations of detrusor and lamina propria were accommodated by the highly coiled structure of collagen in the lamina propria. Imaging and mechanical studies presented here allow gross mechanical response to be attributed to specific components of the bladder wall and further, may be used to investigate the impact of microstructural changes due to pathology or aging, and how they impair tissue functionality. 


\section{Introduction}

The function of the bladder is to store large volumes of urine (typically 400-500 $\mathrm{ml}$ in humans) at low pressure and void stored contents from the body when at capacity. The bladder wall is a complex multi-layer tissue consisting of an outer thick layer of smooth muscle (detrusor) and a loose, fibrous inner layer (lamina propria), allowing the bladder wall to undergo large deformations with minimal resistance during filling and contraction across a great length when voiding. Measurement of the compliance of the bladder during urodynamic studies is used clinically to diagnose lower urinary tract disorders. Although these disorders may affect different layers differentially, the distribution of mechanical load between anatomical layers and microstructural constituents is poorly understood.

The lamina propria is thought to play a capacitance role initially during filling with loose collagen and folded rugae allowing large extension [1]. At higher volumes the flattening of the rugae and straightening of collagen fibrils are supposed to limit distension [2]. The detrusor muscle also plays multiple roles: relaxing during filling and contracting to void urine. The majority of the detrusor consists of bundles of smooth muscle in various orientations, interconnected by collagen fibrils [3]. However, direct evidence of these roles from in situ imaging of the fresh loaded tissue is so far lacking.

The function of the bladder is determined by the mechanical response of the bladder wall, and the extra cellular matrix (ECM) constituents of which it is composed. Alterations to the composition of the bladder wall due to pathology or age are known to affect mechanical response, and thus bladder function, often with severe consequences to patients. Collagens I and III comprise the majority of structural matrix proteins 
throughout the bladder wall. Fibrillar collagen is primarily localised to the lamina propria and surrounding smooth muscle in the detrusor, and arranged in complex coiled microstructures [4]. Chang et al., 1998 speculate that the high compliance of the wall arises from the unfolding and reorientation of multiple 'levels' of smooth muscle before collagen fibrils begin to uncrimp and bear load. However only recently have studies imaged structural proteins in the whole tissue under increments of stretch to observe this directly $[5,6]$.

Second harmonic generation (SHG) and two-photon excitation fluorescence (TPEF) signals from a multiphoton microscope (MPM) provide high resolution imaging of loadbearing proteins collagen and elastin respectively at tissue depths of up to $300 \mu \mathrm{m}$. This permits the use of whole, fresh-frozen tissue with no exogenous stains, avoiding mechanical property and geometric changes due to fixing $[7,8]$.

The aim of this study was to visualize the microstructure of load bearing proteins in the wall layers of the murine bladder, quantify their organization and link whole tissue stressstretch behaviour to microstructural deformation. We hypothesized that the lamina propria would accommodate strains with geometrical rearrangement of first rugae and then fibrils. This aim was achieved using SHG and TPEF MPM imaging, image analysis techniques and in-situ uniaxial mechanical testing of fresh-frozen whole murine bladder tissue together with SHG, TPEF and histological staining of fixed bladder wall crosssections.

\section{Methods}




\subsection{Tissue preparation}

Sixteen, 3-4 month-old male C57/BL6 mice were used in the current study. All experiments were carried out under an approved UK protocol and project license and in accordance with the University of Sheffield Animal Care Committee. Mice were sacrificed by cervical dislocation following isoflurane anaesthesia. Intact bladders were placed in phosphate buffered saline (PBS) and kept frozen at $-20{ }^{\circ} \mathrm{C}$ in excess of 24 hours. The absence of calcium in the storage medium ensured that the bladders contractions were minimized.

Eight bladder specimens were used for mechanical testing and an additional four each used for histology and planar imaging studies. Following thawing for two hours at $23 \pm$ $2^{\circ} \mathrm{C}$ rectangular strips of bladders were cut along the circumferential axis, removing the apex and base regions (See Figure 1). Loose areolar tissue on the outer layer was carefully removed and the specimens were washed in PBS. DAPI stock (Fischer Scientific) was diluted 1:1000 in PBS. Tissue was incubated in DAPI working solution for 10 minutes in dark area, then washed several times with PBS. Full length strips were cut to measure approximately $12 \times 4 \mathrm{~mm}$. Tissue strips were placed in a resting configuration on a microscope cover slip and hydrated with PBS. A second cover slip was placed over the sample to secure the tissue and prevent movement and folding of tissue.

Histology was performed on cross sections excised from the mid-body of bladders $(n=4)$ aligned with the circumferential axis of the bladder. Tissue was fixed in $10 \%$ formalin and processed for paraffin embedding (Leica ASP 300 S, Leica, UK). Microtome 
sections, $10 \mu \mathrm{m}$ thick were cut through the bladder wall thickness and applied to adhesive slides. Tissue samples chosen for staining were rinsed and equilibrated in PBS at room temperature and incubated in DAPI (Fisher Scientific) and thoroughly rinsed with PBS before imaging. Collagen orientation analysis was performed on z-stack maximum intensity projections across the visible cross section of the tissue to obtain orientation across depth.

\subsection{Imaging protocol}

A Zeiss LSM 710 inverted confocal microscope (Zeiss, Oberkochen, Germany) fitted with a pulsed femto- second Chameleon laser operating at $860 \mathrm{~nm}$, pulse width $140 \mathrm{fs}$ at peak and repetition rate $80 \mathrm{Mhz}$ was utilized for imaging urinary bladder tissue. Collagen (SHG) and elastin (TPEF) were imaged at wavelengths of $430 \mathrm{~nm}$ and $520 \mathrm{~nm}$, respectively, with spectral bins of $\pm 20 \mathrm{~nm}$. Objectives used were Zeiss air 10x PlanApochromat (working distance 2mm, NA 0.45), and 20x Plan-Apochromat (working distance $0.55 \mathrm{~mm}, \mathrm{NA} 0.8$ ). For DAPI stained tissues excitation was at $800 \mathrm{~nm}$ and imaging at $460 \mathrm{~nm} \pm 20 \mathrm{~nm}$. Power at the sample with these settings varied from 1500 to $3500 \mathrm{~mW}$. Individual filter channels were overlaid into a single image. Planar images with resolution 1024 x 1024 were acquired over a 5 x 12 tile region and imaged from both inner and outer surface perspectives. A fixed number of optical slices were acquired across different z- stack depths resulting in a z-resolution of between 1 and $5 \mu \mathrm{m}$, depending upon layer imaged and penetration depth. A maximum image height of approximately $200 \mu \mathrm{m}$ was achieved. Cross section images were taken as a tiled region of 5 x 5 images. Bladder wall thickness and width were measured using Image J (W. 
Rasband, NIH, USA).

\subsection{Uniaxial tensile testing protocol}

Mechanical testing data was obtained using a custom in-situ uniaxial system with a linear actuator ( $\pm 1 \mu \mathrm{m}$, Zaber T-NA08A25; Zaber, Vancouver, Canada) and 5N load cell $( \pm 0.5$ $\mathrm{mN}$, Interface, Scottsdale, USA). Rectangular tissue strips $(\mathrm{n}=8)$ were loaded in the device using custom-made clamps, aligning the bladder circumferential axis to the axis of extension. Pre-testing using specimens marked with ink showed no slipping at the clamps for the forces applied. Clamps were submerged in a bath of PBS at ambient temperature and adjusted to achieve a tare load of $0.02 \mathrm{~N}$ after tissue was clamped, and specimen thickness measured with an optical micrometer (mean of five points, Keyence, UK, precision $\left.10^{-5} \mathrm{~m}\right)$.

Bladder strips were subjected to three preconditioning cycles to a stretch of 1.5 with 3 minutes between cycles [9]. Using first Piola-Kirchhoff stress, stress-strain curves were obtained by applying displacement using a triangular ramp function at $1 \% \mathrm{~s}^{-1}$. This was repeated 3 times and results averaged. After the mean stress-strain curve had been recorded, tissue was imaged during uniaxial stretch applied at increments of 0.1, to a maximum stretch limit of 2. Ex vivo inflation experiments [10] suggest this correspondsing approximately to the filling of the bladder from rest to maximum capacity [10]. Stretch was maintained for 2 minutes by which time load cell output had reached a steady value at each step before imaging. Following imaging from one side of the preparation, specimens were flipped and remounted to image both inner and outer surfaces during loading. 


\subsection{Collagen orientation analysis}

Collagen orientation through the wall thickness was measured in each 1024 x 1024 image in the $200 \mu \mathrm{m}$ deep MPM SHG z-stack, obtained with the detrusor side facing the objective. An algorithm in Matlab (Mathworks, Natick, MA) implemented a 2D fast Fourier transform method [11]. This converts spatial patterns into frequency components with a directional dependency enabling orientation magnitudes from 0 to $180^{\circ}$ to be calculated. A raised cosine window was applied to minimise frequency domain errors due to periodic discontinuities caused by image boundaries. Discrete orientation data were obtained using a wedge filter.

\section{Results}

\subsection{Imaging of planar sections}

Combined maximum intensity projections over a depth of $20 \mu \mathrm{m}$ of MPM images of the luminal side of the wall (Fig 2) reveal a distinct stromal layer (Fig 2, left top) with a fine, densely interwoven network of collagen on top of a deeper, sparser more isotropic tissue with larger fibril bundles (Fig 2, left bottom). In the lower magnification image (Fig 2 right) folds in the rugae appear as dark, out of plane regions, mainly aligned longitudinally.

Highly aligned discrete bundles of collagen fibrils are seen in the detrusor layer (Fig 3, right) orientated in both circumferential (horizontal) and longitudinal (vertical) axes. Abrupt changes in collagen fibril bundle orientation between perpendicular bundles of 
collagen suggest these bundles occupy layers at different depths through the tissue. A tortuous blood vessel was seen across the longitudinal axis in the detrusor layer. Higher magnification (Fig 3, left top and bottom) shows straight fibrils within discrete aligned bundles.

\subsection{Cross section imaging}

A representative bladder cross section shows a fine, dense collagen layer on the luminal surface 8 - $18 \mu \mathrm{m}$ thick (SHG, green, Fig 4a). This is underlaid by larger, undulated fibril bundles sparsely localised throughout the lamina propria. The folded rugae give the lamina propria a highly variable thickness. Approximately 320 - $780 \mu \mathrm{m}$ from the luminal surface (measured from peaks of rugae) a clear transition can be distinguished between the lamina propria and detrusor (Fig 4a, arrow).

The detrusor forms the majority of the bladder wall, with a thickness of approximately $480-770 \mu \mathrm{m}$. Elastin is present in the detrusor layer and serosa, conforming to the orientation of smooth muscle bundles visualised by DAPI staining nuclei (TPEF, red, Fig 4a,b). Smaller collagen fibrils interweave the smooth muscle bundles in the detrusor both longitudinally and circumferentially (Fig 4c). Frequently, between muscle bundles empty regions are seen forming circumferential and longitudinal 'pockets'.

\subsection{Collagen orientation in the detrusor}

The orientation algorithm was used with combined SHG and DAPI signals across the wall thickness of urinary bladders $(n=4)$ in an unloaded state, starting from the detrusor side (Fig 5a \& e). DAPI staining of the rod-like smooth muscle nuclei indicates muscle 
orientation. Nuclei align with orientation of collagen fibrils throughout the depth of the detrusor (Fig 5b,c,d,f,g\&h). Following a thin adventitial layer (Fig 5d \& h), consisting largely of loose collagen fibril bundles, the first layer of smooth muscle is observed 20$40 \mu \mathrm{m}$ from the outer surface (Fig $5 \mathrm{c} \& \mathrm{~g}$ ). The initial muscle layer occupies approximately 50-90 $\mu \mathrm{m}$ of the wall, and is oriented roughly circumferentially. Deeper to this, bundle orientation changes abruptly through approximately $90^{\circ}$ defining a thicker longitudinal muscle layer (Fig 5b\&f). These observations are consistent with two well-defined families of bundles aligned approximately along the longitudinal and circumferential axes of the bladder at different depths through the wall. Images also confirm high cell density in the detrusor layer and comparatively sparse cellularity of the lamina propria.

\subsection{Uniaxial tensile testing and in situ MPM}

The luminal surface of the bladder tissue strips is imaged using MPM under incremental stretch in a uniaxial testing device (Fig 6a). No evidence of failure at stretches of up to 2 is seen in force-displacement data, nor in the macro- and microscopic integrity of the specimen.

Initially, large undulations of the rugae are seen with a fine, dense collagen network forming the surface. At a stretch of 1.4 rugae begin to flatten and at 1.6 have almost completely flattened. Collagen at this stretch remains loose and isotropic. At a stretch of 2 the rugae are entirely flat and collagen re-orientates in the direction of loading. Fibrils straighten uniformly.

In the detrusor layer fibril groups initially appear straight with a local alignment specific 
to each bundle (Fig 6b) and retain their straightness as stretch increases to 1.4. Multiple grouped bundles of fibrils become visible in the same plane. Fibrils begin to re-orientate in the direction of loading at a stretch of 1.6. A stretch of 2 shows fibrils becoming thinner under tension.

Plotting the stress-stretch response of murine bladder together with 3D visualisation of MPM enables identification of microstructural deformation mechanisms leading to its highly non-linear behaviour (Fig 7). These images identify two regions in the compliant toe region: long wavelength rugae unfolding first $(0<\lambda<1.2)$ followed by shorter wavelength rugae $(1.2<\lambda<1.6)$. In the heel region $(1.6<\lambda<1.8)$ a significant increase in stiffness occurs, coinciding with complete flattening of the rugae. Further stretch $(\lambda>$ 1.8) shows increased stiffening of the tissue and with collagen realigning in the direction of loading and straightening completely.

\section{Discussion}

Using MPM imaging and in situ uniaxial mechanical testing for the first time in whole, fresh-frozen murine bladder tissue this study demonstrates the anatomical layer specific differences in collagen morphology, orientation and response to uniaxial loading. This confirmed the hypothesis that the lamina propria deformed first by unfolding of the rugae followed by rearrangement of fibrils. The detrusor also showed fibril rearrangement at lower strains, Further, image analysis quantification of collagen and smooth muscle organisation confirmed two families of smooth muscle and collagen bundles with a thinner, shallow circumferential layer and a deeper, thicker longitudinal layer. SHG microscopy showed the lamina propria to have two distinct layers: a thin, collagen dense 
stromal layer of fine collagen on the inner lumen and a thicker layer of sparse, highly coiled large collagen fibres. Finally, tissue sections confirmed this highly non-uniform distribution of collagen and elastin and the presence of 'pockets' in the tissue empty of structural load bearing proteins.

The undulated geometry of the rugae require large strains to unfold and become taut before the layer begins to bear tension. In contrast, the detrusor rests in an unfolded state that follows the perimeter of the bladder wall, so collagen and muscle bear load. The result of these two very different resting reference configurations means the two layers are subjected to different stretches during whole wall expansion (Figure 8). The highly coiled collagen of the lamina propria may accommodate for these different stretch configurations without causing shear stress at the layer interface and allowing the rugae to unfold with minimal resistance.

Previously microscopy of the bladder in a loaded configuration was carried out on snap frozen foetal calf bladders inflated to three or four different volumes up to $100 \%$ capacity [2]. They proposed that the lamina propria was already load bearing between $0 \%$ and $25 \%$ of maximum capacity. Figure 7 shows this layer thinning rapidly, however it retains a loose appearance indicating it is unlikely to have the structural integrity to bear tension until higher stretches of approximately 1.6. Disproportionate thinning of the lamina propria may be a result of rugae flattening and unfolding rather than stress-thinning as a result of bearing tension in the wall.

MPM images of bladder cross sections in Figure 4 show large regions of free space in the detrusor between discrete muscle bundles. Tortuous collagen fibrils sparsely occupy 
'pockets' in the detrusor and appear to attach nearby muscle bundles. A possible function of these pockets is to allow independent muscle bundles in both axes to extend fully and may allow a degree of sliding between layers of muscle as the bladder wall thins during distension (Fig 7). At higher stretches collagen uncoils; hypothesized to limit muscle bundle separation and act as a girding mechanism to limit expansion and stop overdistension. This was also observed by Chang et al., 1998 who reported that collagen type III fibres uncoil in the detrusor during distension and become fully straightened at $100 \%$ capacity.

Collagen and smooth muscle orientation was quantified across the depth of the bladder wall. The orientation and interaction between smooth muscle and collagen is important in understanding the bladder as they underlie passive and active mechanical response, and geometry [12]. MPM imaging of the detrusor shows an outer layer of collagen and smooth muscle orientates circumferentially, and a second deeper layer with longitudinal alignment. This arrangement was seen in cross section images in Figure 4 as long bands of collagen and muscle occupy the outer detrusor and cross sections of longitudinal muscle bundles near the lamina propria. Previously, polarised light imaging showed the bladder wall to be highly anisotropic and heterogeneous with anisotropy increasing at increased distension [13]. A possible explanation for this may be collagen losing its coiled form and aligning more towards its specific orientation.

The stress-stretch response of murine bladder wall has a long toe region. During this phase the smooth muscle elongates, unfolds and reconfigures; while collagen was observed to play a capacitance role in a coiled state. At low stretches rugae of the inner lumen retain a wavy appearance; suggesting they lack the form to bear loading. The heel 
region of the stress-stretch response occurs with flattening of rugae and collagen reorientation in the direction of loading. Additionally, a majority of collagen in the detrusor straightens, suggesting the detrusor is load bearing. It was not until the linear portion of the stress-stretch curve that the rugae are seen to flatten and collagen straightens in the lamina propria. This mechanism may play a distension limiting role in conjunction with the detrusor. The high collagen content and large fibril bundle size provides a structural means to bearing larger loads.

The uniaxial test technique does not reflect physiological loads on the tissue and is associated with non-affine fibril kinematic behaviour where biaxial can be modelled as affine [14]. Despite this, the large extent of coiling suggests that the coiled fibres capacitance role would be maintained under biaxial load but engaged at lower strains due to the higher level of constraint. Detrusor pocket features observed may be artefacts of highly non-affine kinematics under uniaxial loading.

This study has other limitations. Frozen specimens were used and although ultrastructural changes have been observed following freeze-thaw cycles no significant differences in mechanical response with fresh material were observed in multi-scale studies in other soft tissues [16-18]. Cutting the bladder and opening out into flat strips will relieve any residual stress and introduce other small, non-physiological deformations. In this study zero strain was defined by a tare load of $0.02 \mathrm{~N}$, however in vivo data are lacking enabling this to be related directly to the 'at rest' bladder configuration. Mouse bladder is increasingly used as a model for human disease [19]. However in the present study the material was approximately an order of magnitude more compliant than uniaxially tested human material [20], although unreported details on tare load and stress calculation make 
comparison difficult. Due to technical challenges there are as yet no reported studies using SHG to observe fibril recruitment in intact human bladder however biaxial loading is a topic of ongoing research in the group [15].

In this study only the inactive bladder wall was characterised, but smooth muscle activation regulates tone during filling and provides active contraction during voiding. A comparison of passive and inactive states of rat bladder by Parekh et al., 2010 showed lower overall compliance in a passive state and significantly higher peak pressures [21]. Nagatomi et al., 2008 also note removal of smooth muscle from rat bladder significantly reduces viscoelasticity [9]. Stress-stretch curves produced here may therefore differ from the behaviour expected in vivo.

The novel information in this work on microstructural deformation and the differential mechanical role of tissue layers will assist in sophisticating models of the bladder used in the interpretation of clinical urodynamics models. Simple mechanical models of micturition proposed so far $[22,23]$ do not capture specific tissue features such as fibrotic change or changes due to ageing, and a microstructurally motivated model could offer improved sensitivity and specificity for diagnosing associated conditions.

\section{Conclusions}

Reporting the first in-situ MPM imaging of uniaxial loaded bladder tissue, this study shows the very different structural roles of the lamina propria and detrusor. The lamina propria deforms with unfolding of the rugae and rearrangement of collagen fibrils at low strains. Further, the highly coiled collagen in the lamina propria accommodates a wide 
range of stretch configurations, potentially enabling large bladder volume changes without shear failure at the interface with the detrusor. The detrusor appears to accommodate large changes in volume by coiled collagen connecting smooth muscle cells, allowing bundles to slide across each other while pockets allow for movement and relocation of collagen. At high stretches, the detrusor contributes to a girding structure to limit distension. The new understanding reported here of the mechanical role and microstructural arrangement of the layers of the bladder will in future enable richer information to be extracted from urodynamic data. New computational bladder models, simulating mechanical behaviour at microstructural, tissue and organ levels, will be used to interpret these data, allowing the accurate quantification of bladder dysfunction. Further, mechanobiological models will be used to predict the evolution of bladder disease and the outcomes of interventions, and by guiding understanding will lead to more effective and safer urology care by generating new options for diagnosis and management of patients with bladder dysfunction.

\section{Acknowledgements}

Jack Hornsby's studentship is supported by the RCUK Digital Economy Programme grant number EP/G036861/1 (Oxford Centre for Doctoral Training in Healthcare Innovation). We would like to thank Dr. Clarence Yapp (University of Oxford, UK) for providing microscope training and expertise. Tissue samples collected and provided by SHaRM, UK (Dr. Daniel Bolland (Babraham Institute, UK) and Dr. Adele Duran (ShARM, UK)) is gratefully acknowledged and appreciated. Rosetrees Trust (M186-F1) are acknowledged for their generous support of this project. Paul Watton acknowledges 
partial support towards this work from the UK EPSRC (EP/N014642/1).

\section{References}

[1] D.H. Ewalt, P.S. Howard, B. Blyth, H.M. Snyder, 3rd, J.W. Duckett, R.M. Levin, E.J. Macarak, Is lamina propria matrix responsible for normal bladder compliance?, J Urol 148(2 Pt 2) (1992) 544-9.

[2] S.L. Chang, J.S. Chung, M.K. Yeung, P.S. Howard, E.J. Macarak, Roles of the lamina propria and the detrusor in tension transfer during bladder filling, Scand J Urol Nephrol Suppl 201 (1999) 38-45.

[3] F. Koenig, S. Gonzalez, W.M. White, M. Lein, M. Rajadhyaksha, Near-infrared confocal laser scanning microscopy of bladder tissue in vivo, Urology 53(4) (1999) 853-7.

[4] S.L. Chang, P.S. Howard, H.P. Koo, E.J. Macarak, Role of type III collagen in bladder filling, Neurourol Urodyn 17(2) (1998) 135-45.

[5] F. Cheng, A.M. Robertson, L. Birder, F.A. Kullmann, J. Hornsby, P. Watton, S.C. Watkins, An experimental approach for understanding layer dependent role of collagen recruitment during loading in the rat bladder wall, in: P. Nithiarasu, A.M. Robertson (Eds.) 5th International Conference on Computational and Mathematical Biomedical Engineering, Pittsburgh, PA, 2017, pp. 396-399.

[6] J. Hornsby, Bladder Microstructural and Biomechanical Modelling: in Vivo, in Vitro and in Silico, Department of Engineering Science, University of Oxford, 2016.

[7] G. Cox, E. Kable, Second-harmonic imaging of collagen, Methods Mol Biol 319 (2006) 15-35. 
[8] M.R. Hill, X. Duan, G.A. Gibson, S. Watkins, A.M. Robertson, A theoretical and nondestructive experimental approach for direct inclusion of measured collagen orientation and recruitment into mechanical models of the artery wall, J Biomech 45(5) (2012) 762-71.

[9] J. Nagatomi, K.K. Toosi, M.B. Chancellor, M.S. Sacks, Contribution of the extracellular matrix to the viscoelastic behavior of the urinary bladder wall, Biomech Model Mechanobiol 7(5) (2008) 395-404.

[10] F. Ronchi, V.E. Pricolo, L. Divieti, M. Palmi, L. Brigatti, G.M. Clement, Experimental study on bladder wall's strain in vesical function, Urol Res 10(6) (1982) 285-91.

[11] A.J. Schriefl, H. Wolinski, P. Regitnig, S.D. Kohlwein, G.A. Holzapfel, An automated approach for three-dimensional quantification of fibrillar structures in optically cleared soft biological tissues, J R Soc Interface 10(80) (2013) 20120760. [12] K.E. Andersson, A. Arner, Urinary bladder contraction and relaxation: physiology and pathophysiology, Physiol Rev 84(3) (2004) 935-86.

[13] S. Alali, K.J. Aitken, A. Schroder, D.J. Bagli, I. Alex Vitkin, Optical assessment of tissue anisotropy in ex vivo distended rat bladders, J Biomed Opt 17(8) (2012) 086010.

[14] T.W. Gilbert, M.S. Sacks, J.S. Grashow, S.L. Woo, S.F. Badylak, M.B. Chancellor, Fiber kinematics of small intestinal submucosa under biaxial and uniaxial stretch, J Biomech Eng 128(6) (2006) 890-8.

[15] F. Cheng, A.M. Robertson, J. Hornsby, L. Birder, S. Watkins, P. Watton, An experimental approach for understanding the layer dependent role of collagen 
recruitment during loading in the rat bladder wall, in: P.N.a.A. Robertson (Ed.) 5th International Conference on Computational and Mathematical Biomedical Engineering - CMBE2017, Pittsburgh, PA, 2017, pp. 396-399.

[16] A. Hopkinson, V.A. Shanmuganathan, T. Gray, A.M. Yeung, J. Lowe, D.K. James, H.S. Dua, Optimization of amniotic membrane (AM) denuding for tissue engineering, Tissue Eng Part C Methods 14(371-81) (2008).

[17] I. Prasertsung, S. Kanokpanont, T. Bunaprasert, V. Thanakit, S. Damrongsakkul, Development of acellular dermis from porcine skin using periodic pressurized technique, J Biomed Mater Res B Appl Biomater 85 (2008) 210-9.

[18] G. Fessel, Y. Li, V. Diederich, M. Guizar-Sicairos, P. Schneider, D.R. Sell, V.M. Monnier, J.G. Snedeker, Advanced glycation end-products reduce collagen molecular sliding to affect collagen fibril damage mechanisms but not stiffness, PLoS One 9(11) (2014) e110948.

[19] C. Seager, A.M. Puzio-Kuter, C. Cordon-Cardo, J. McKiernan, C. Abate-Shen, Mouse models of human bladder cancer as a tool for drug discovery, Curr Protoc Pharmacol Chapter 14 (2010) Unit14 14.

[20] P.A. Martins, A.L. Filho, A.M. Fonseca, A. Santos, L. Santos, T. Mascarenhas, R.M. Jorge, A.J. Ferreira, Uniaxial mechanical behavior of the human female bladder, Int Urogynecol J 22(8) (2011) 991-5.

[21] A. Parekh, A.D. Cigan, S. Wognum, R.L. Heise, M.B. Chancellor, M.S. Sacks, Ex vivo deformations of the urinary bladder wall during whole bladder filling: contributions of extracellular matrix and smooth muscle, J Biomech 43(9) (2010) 1708-16. 
[22] A.S. Paya, D.R. Fernandez, D. Gil, J.M. Garcia Chamizo, F.M. Perez, Mathematical modelling of the lower urinary tract, Comput Methods Programs Biomed 109(3) (2013) 323-38.

[23] F.A. Valentini, G.R. Besson, P.P. Nelson, P.E. Zimmern, A mathematical micturition model to restore simple flow recordings in healthy and symptomatic individuals and enhance uroflow interpretation, Neurourol Urodyn 19 (2000) 153176.

\section{Competing Interests}

We have no competing interests

\section{Author Contribution}

JH designed the study, carried out experimental and computational work and drafted the manuscript. DD aided in experimental studies and with DG provided animal tissue and contributed to the manuscript draft. AR contributed with consulting and drafting the manuscript. PW and MT supervised JH and aided with design of the study, manuscript drafting and coordinated resources. 


\section{Author Funding}

Jack Hornsby - RCUK CDT in HI (EP/G036861/1), Rosetrees M186-F1. Paul Watton EPSRC (EP/N014642/1). 
Figure 1: Illustration of location and dimensions of strips dissected from mouse bladders. Axes $\mathrm{x}$ and y correspond to circumferential and longitudinal directions, respectively.

Figure 2: MPM images of collagen (SHG, green) in the luminal murine bladder wall. Overview of complete body (right). Detailed views of organised and regular mesh of collagen in the stroma (top-left), and looser, more randomly aligned collagen in the lamina propria (bottom-left). Axes $\mathrm{x}$ and y correspond to circumferential and longitudinal orientation, respectively.

Figure 3: MPM images of collagen (SHG, green) in the detrusor across the body of the bladder wall (right). Detailed views of highly aligned collagen 'bundles' overlapping in the detrusor (left). Arrow indicates blood vessel in the wall.

Figure 4: MPM images of murine bladder wall cross sections. (a) overview of collagen (SHG, green) and enhanced contrast elastin (TPEF, red) with arrow indicating interface between detrusor and lamina propria. (b) cell nuclei (DAPI, blue) and (c) detail of collagen in the detrusor, * indicating 'pocket' feature. LP and Det indicate lamina propria and detrusor, respectively. Arrows in (b) and (c) indicate direction of bladder wall circumference.

Figure 5: (a and e) Normalised orientation magnitude plot calculated from combined MPM collagen (SHG, green) and smooth muscle cell nuclei (DAPI, blue) images across the wall depth imaged from the surface of the detrusor in two bladders. Orientations of $90^{\circ}$ correspond to circumferential axis, $0^{\circ}$ and $180^{\circ}$ correspond to the longitudinal axis. Red and yellow regions indicate strong alignment, identifying two bundle families i) shallow, longitudinal ii) deep circumferential. MPM images at depths of (b and f) $150 \mu \mathrm{m}$ (c and g) 20-40 $\mu \mathrm{m}$ and ( $\mathrm{d}$ and $\mathrm{h}$ ) the surface of the detrusor.

Figure 6: MPM images of collagen in a) the lamina propria surface and b) detrusor layers under incremental uniaxial stretch. Arrows indicate visible lateral thinning of fibre bundles in the detrusor.

Figure 7: Uniaxial first Piola-Kirchhoff stress-stretch curves for murine bladders $(\mathrm{n}=4)$ with corresponding 3D reconstructions (c. 800 x $800 \times 200 \mu \mathrm{m}$ ) of representative MPM images of the luminal surface of the lamina propria at stretches of (a) 1, (b) 1.2, (c) 1.6, (d) 2

Figure 8: Schematic of the initial configurations and relative stretch of the rugae and detrusor showing illustration of smooth muscle and collagen changing states from empty to distended. Collagen acts as a capacitance mechanism to allow smooth muscle reconfiguration and elongation and a limiting 'girding' to prevent over-distension. $\lambda_{\mathrm{LP}}$ and $\lambda_{\text {Det }}$ are stretches in the lamina propria and detrusor, respectively (assuming $\lambda=1$ is the point at which the layer unfolds). It is hypothesised highly coiled collagen in the lamina propria accommodates the different stretch configurations and minimises shear 
between layers. 

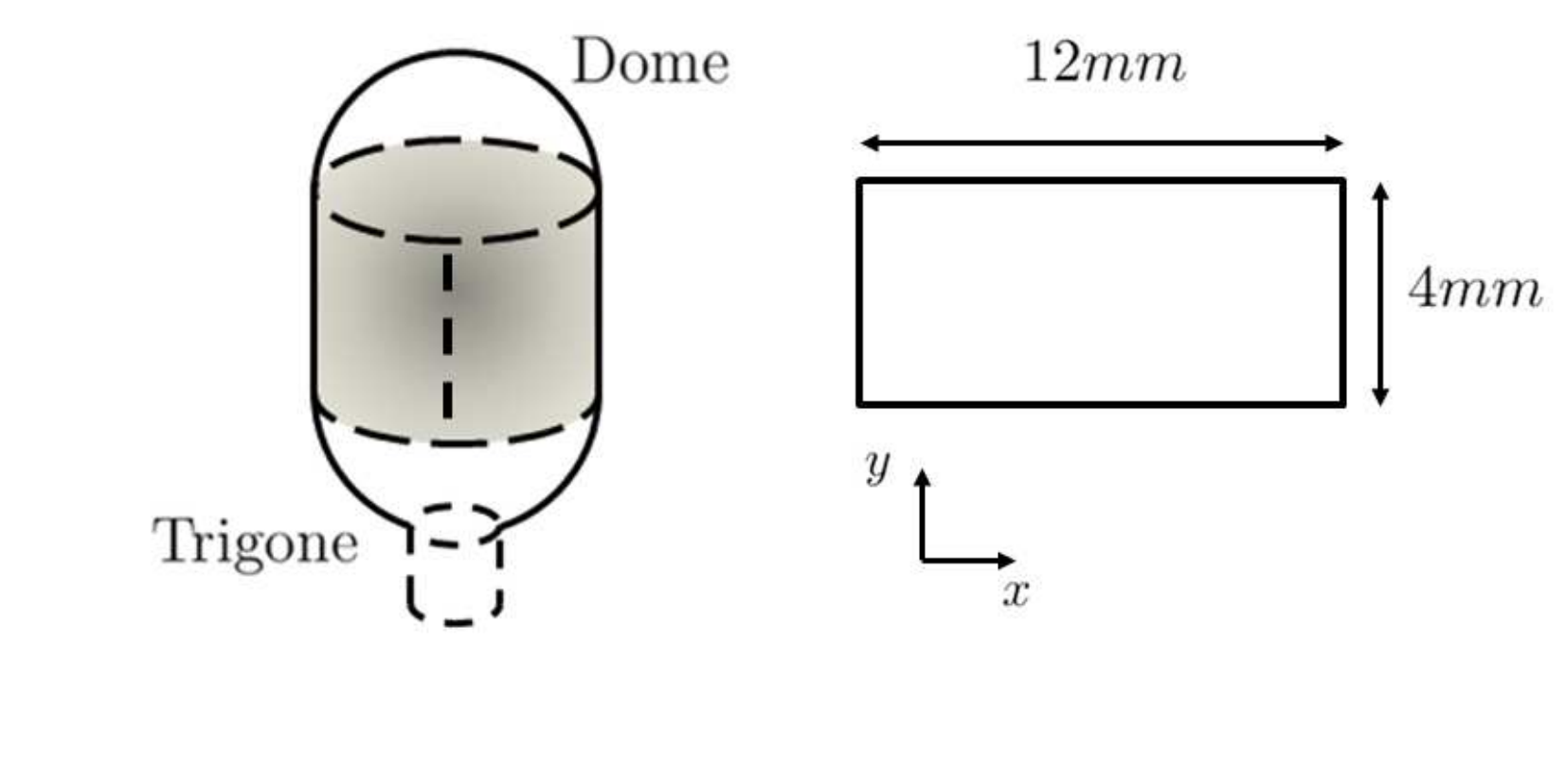

Figure 1

$$
\text { Le }
$$

$12 \mathrm{~mm}$

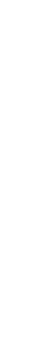
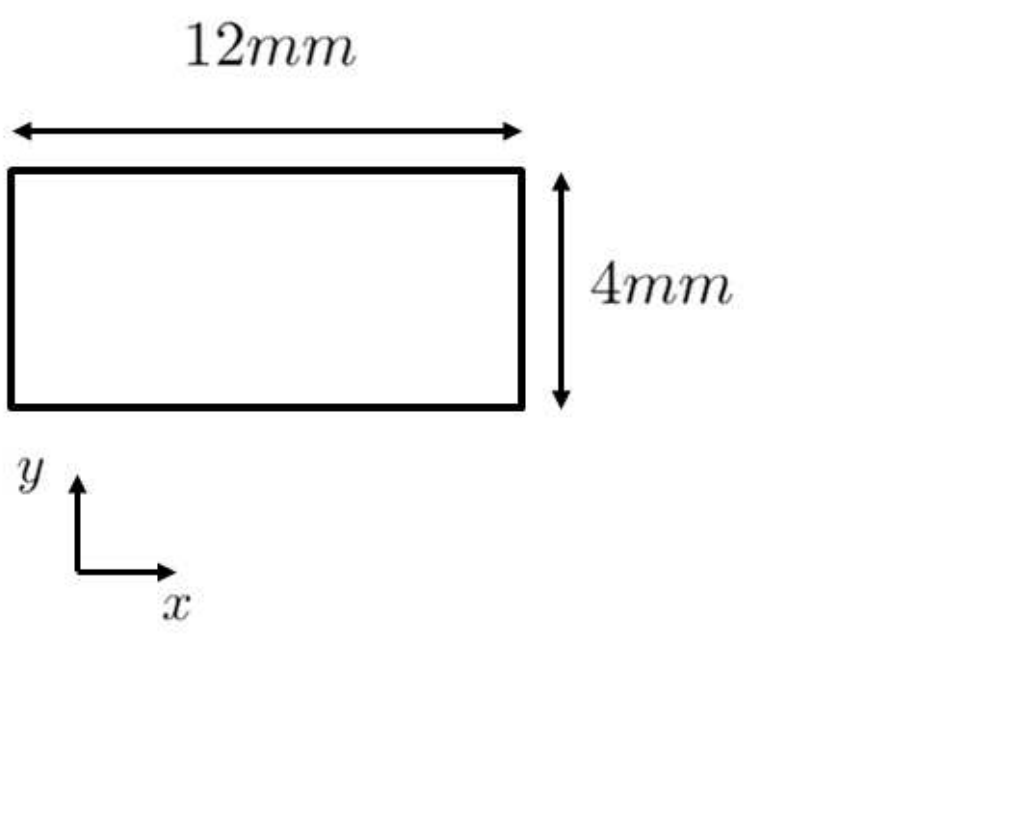


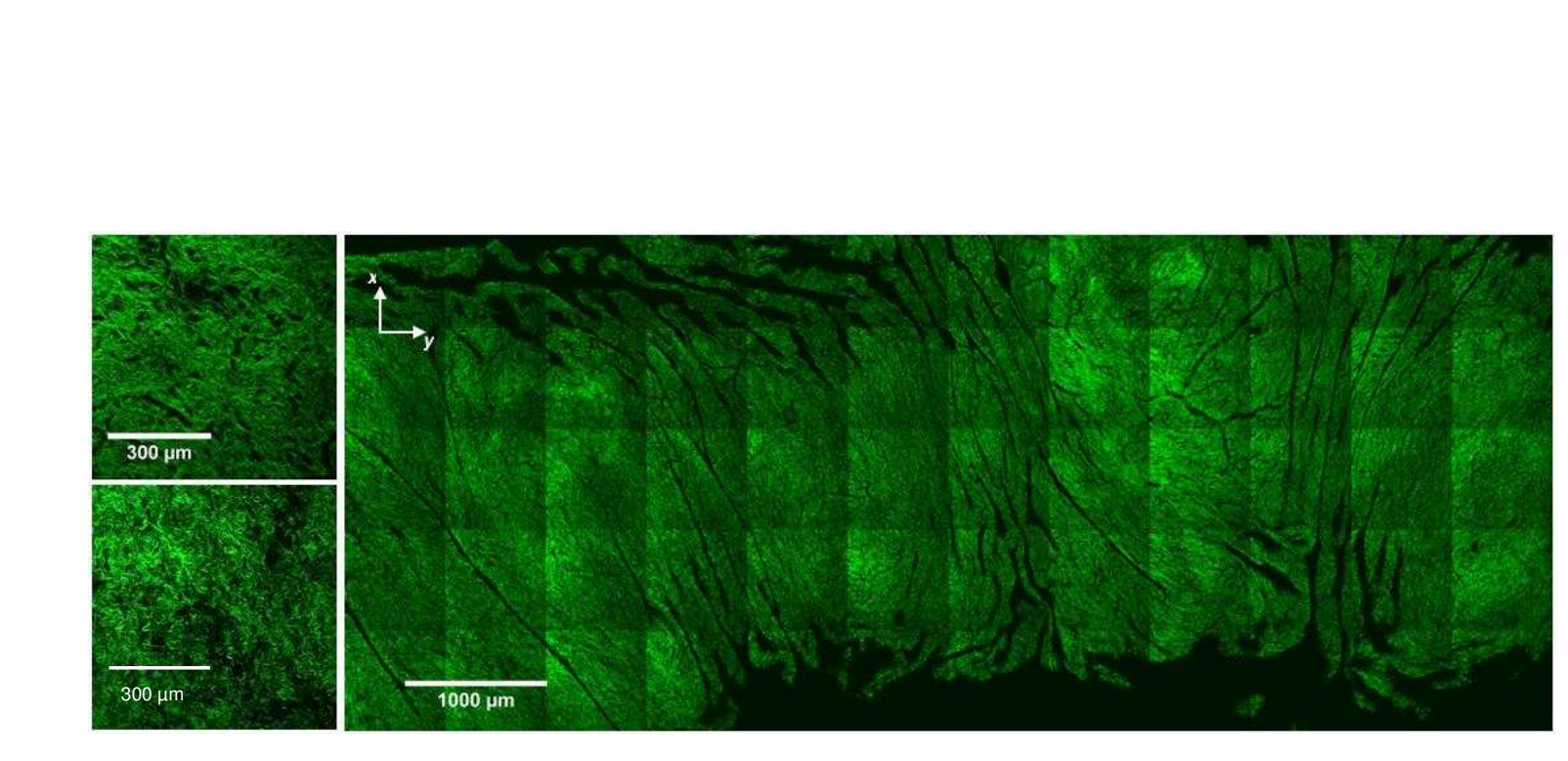

Figure 2

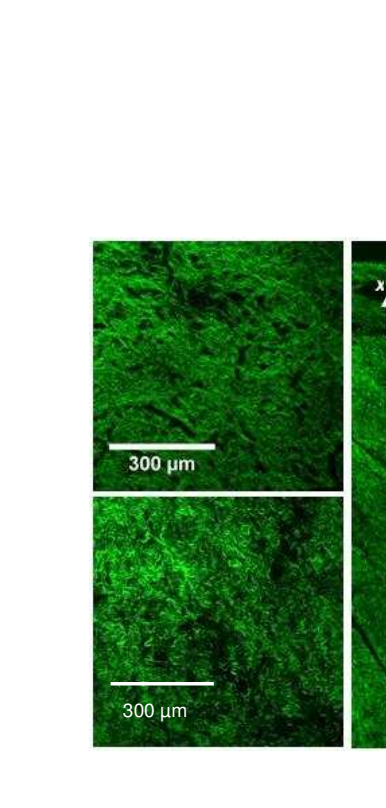

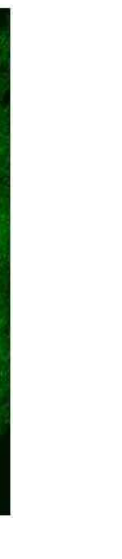
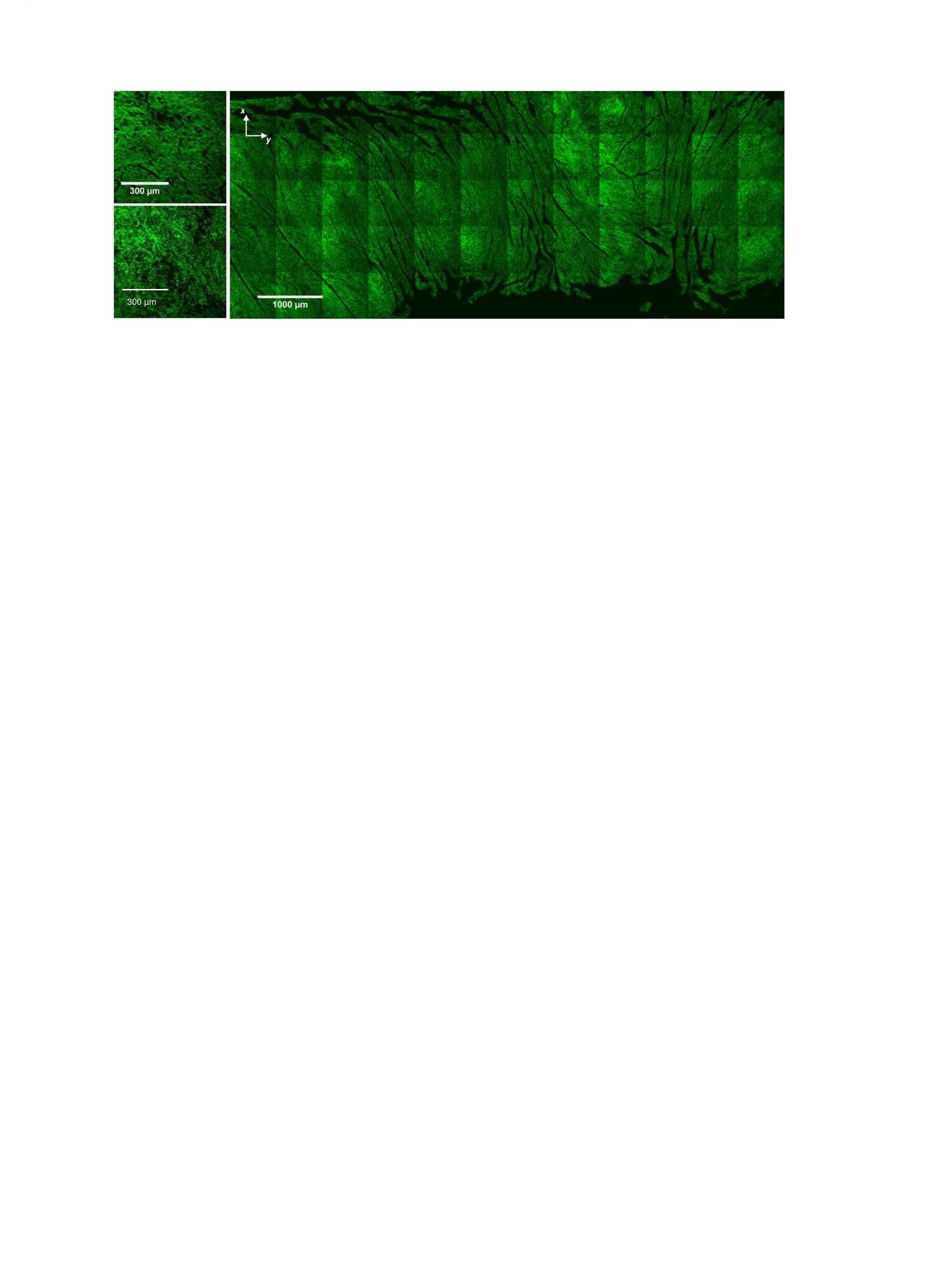


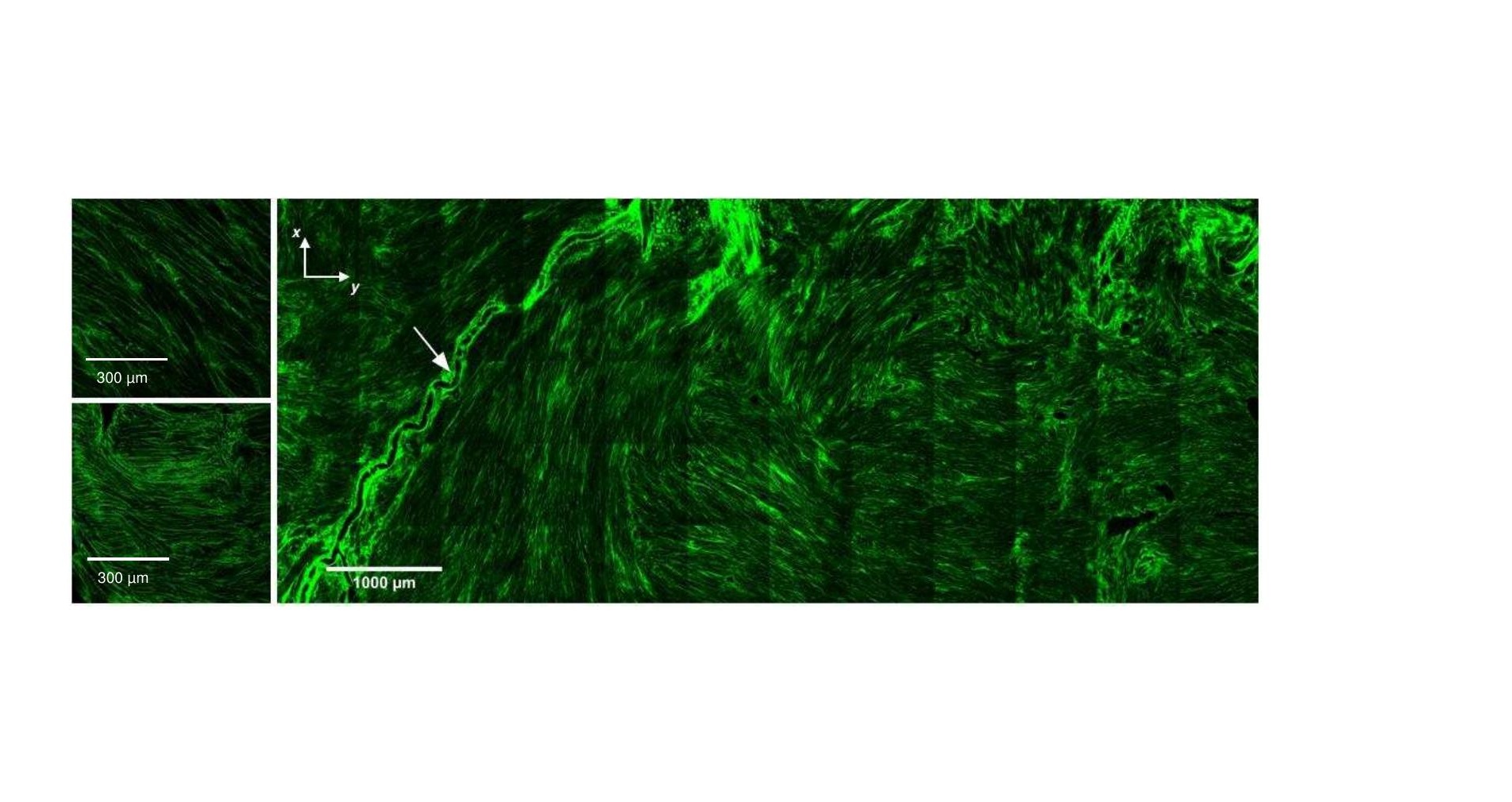

Figure 3

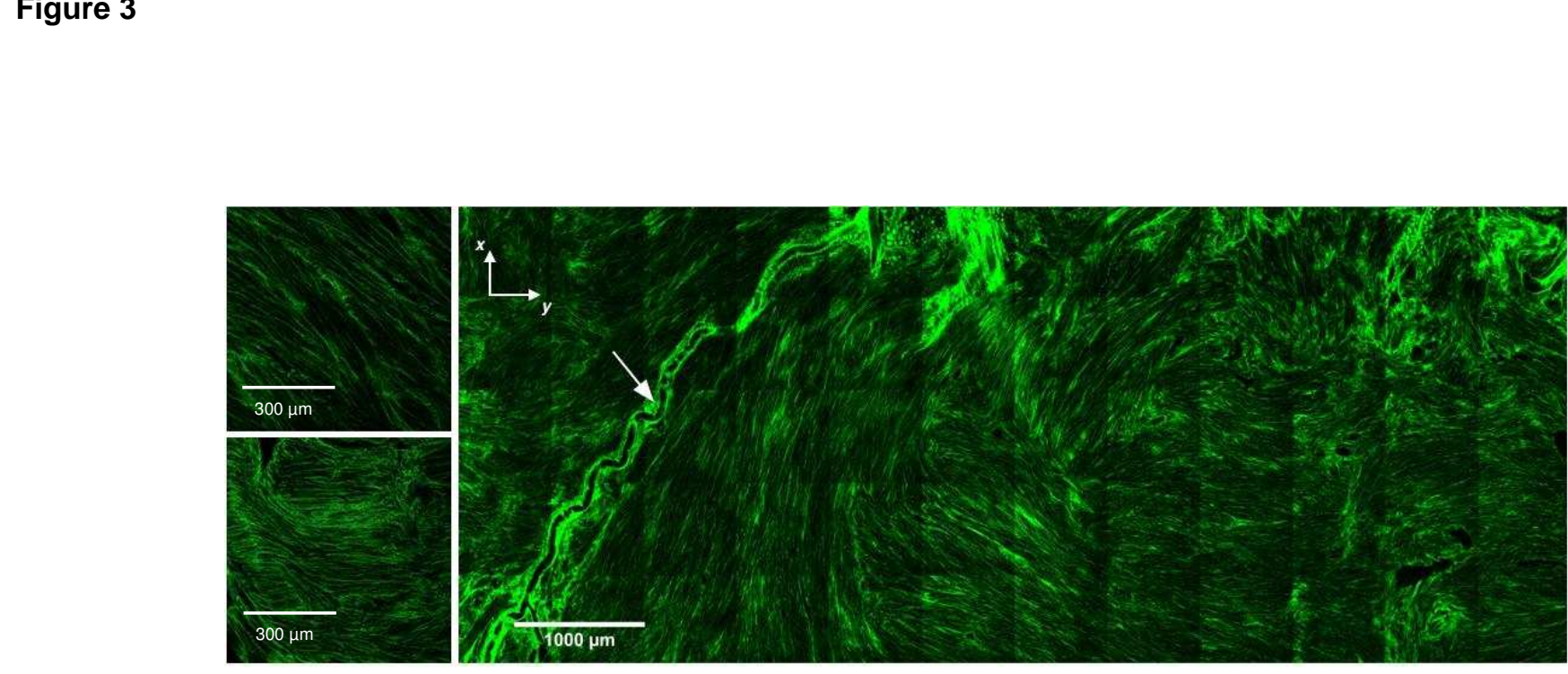

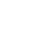
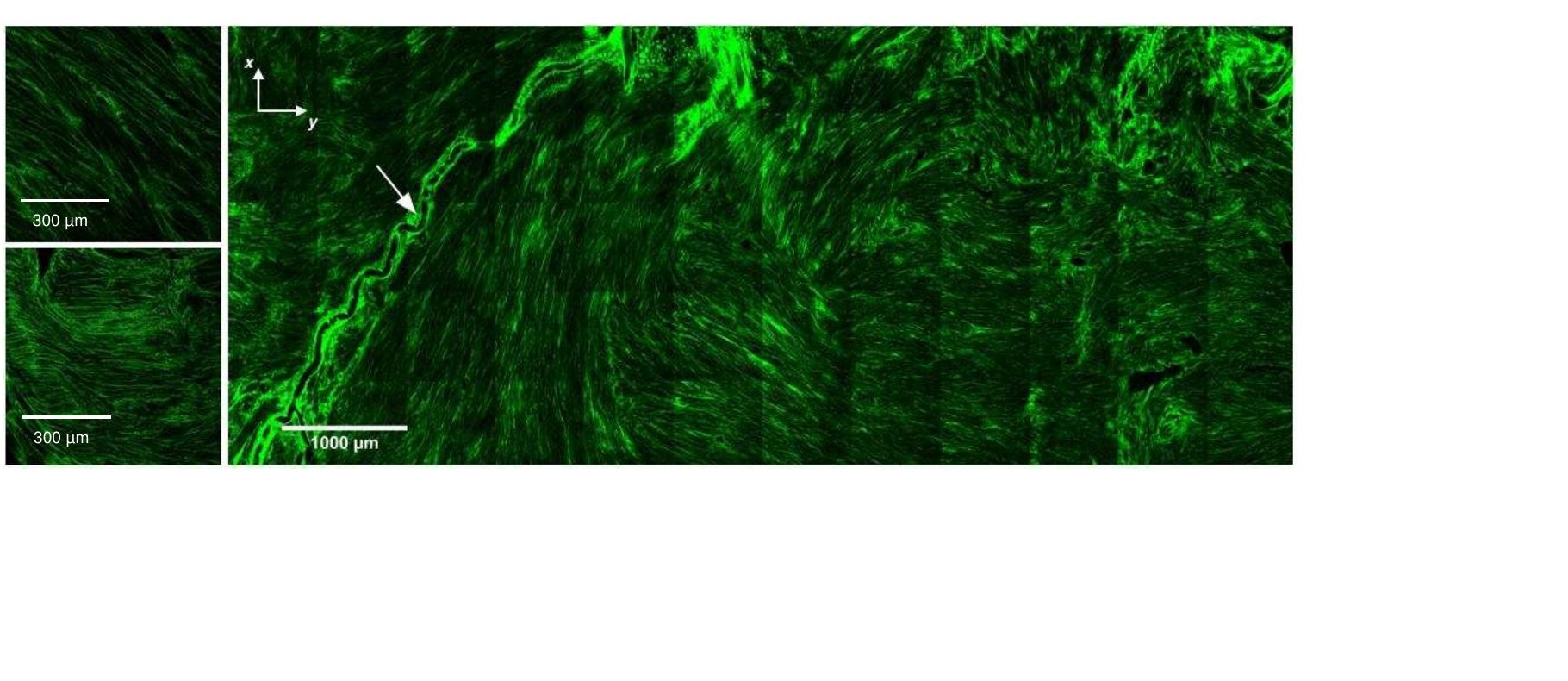

.
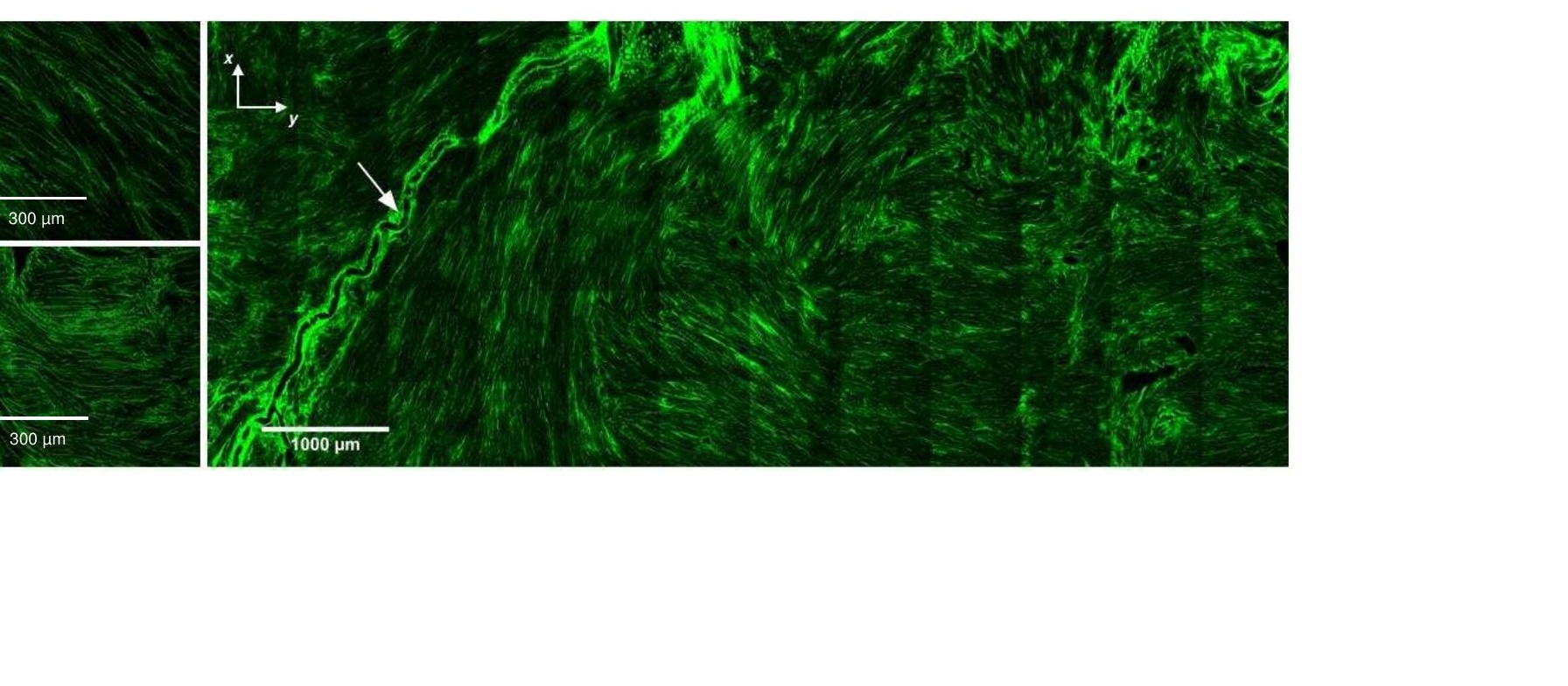

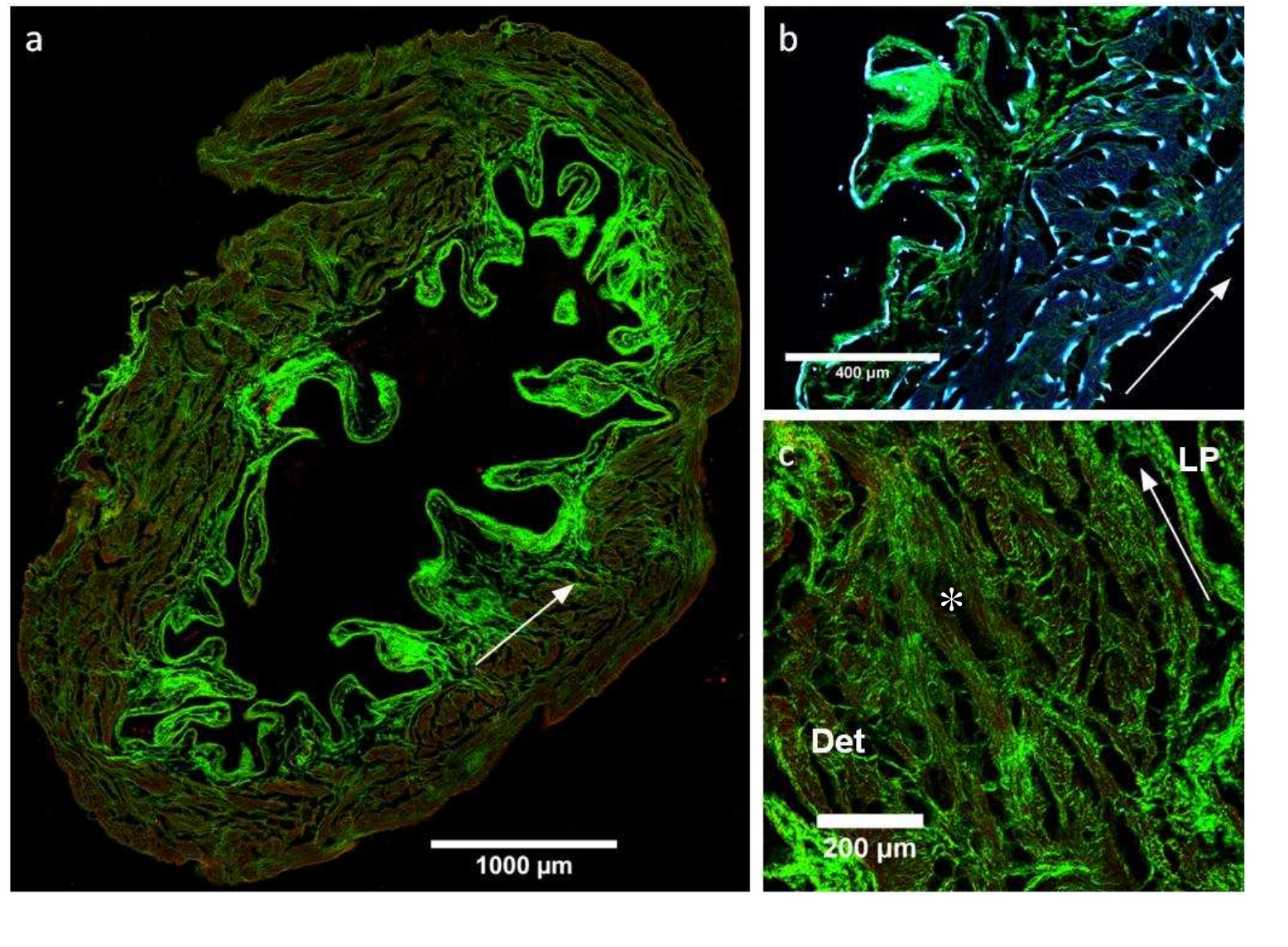

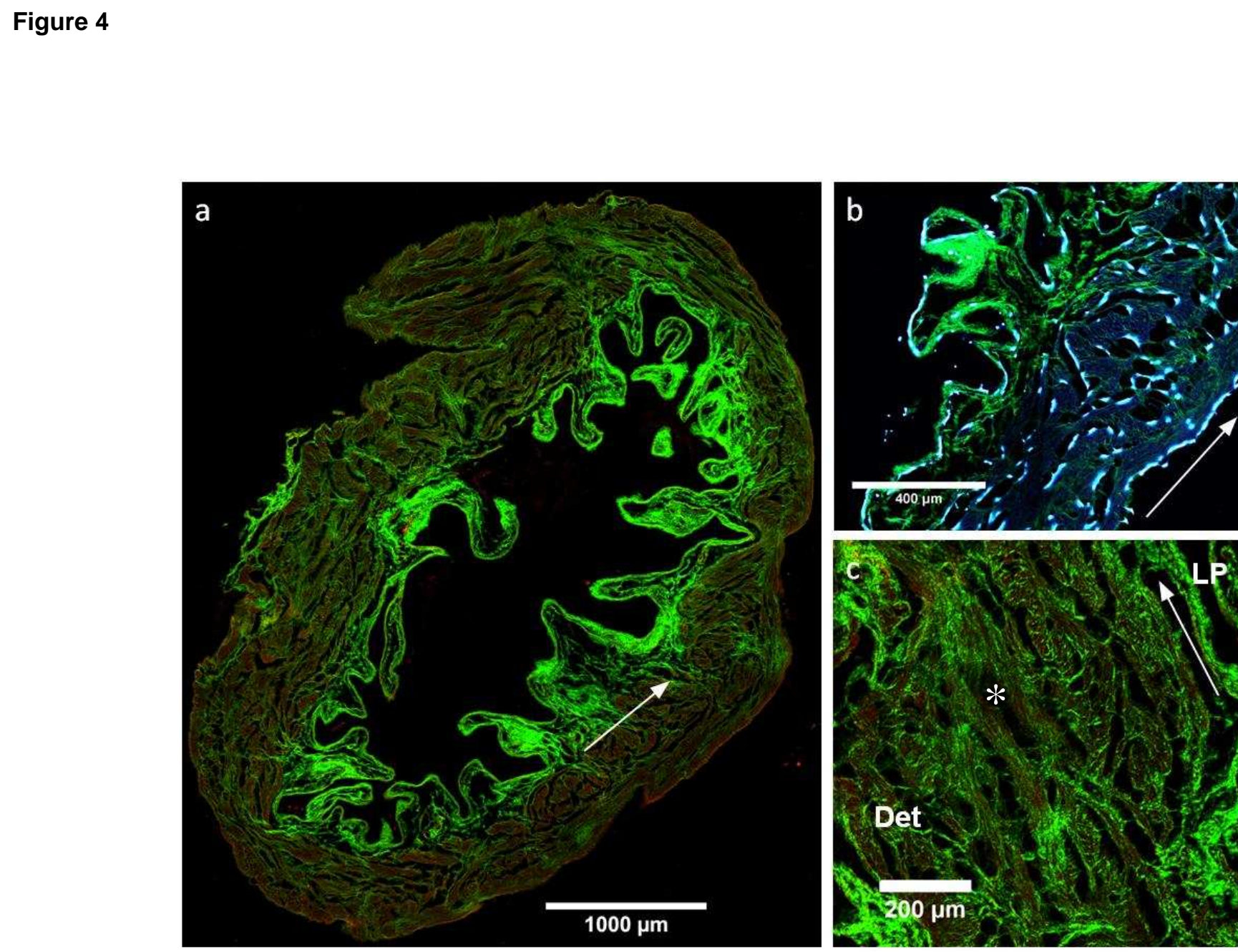

(⿸丆口
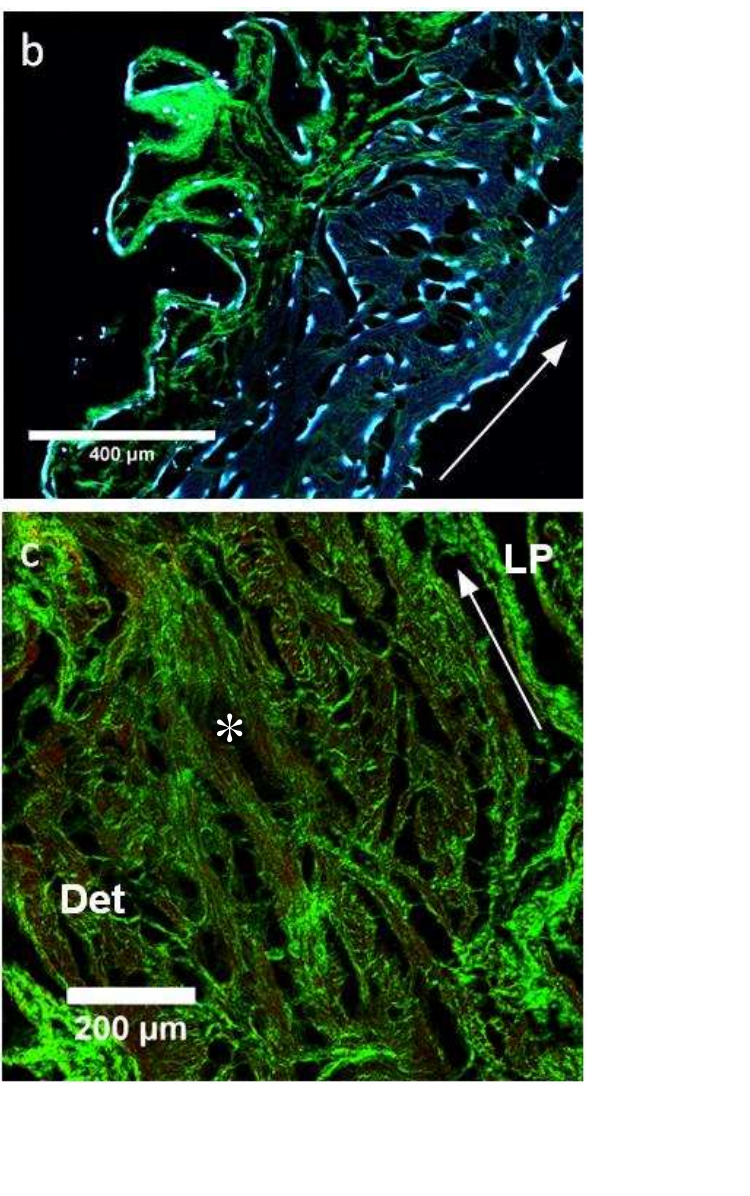

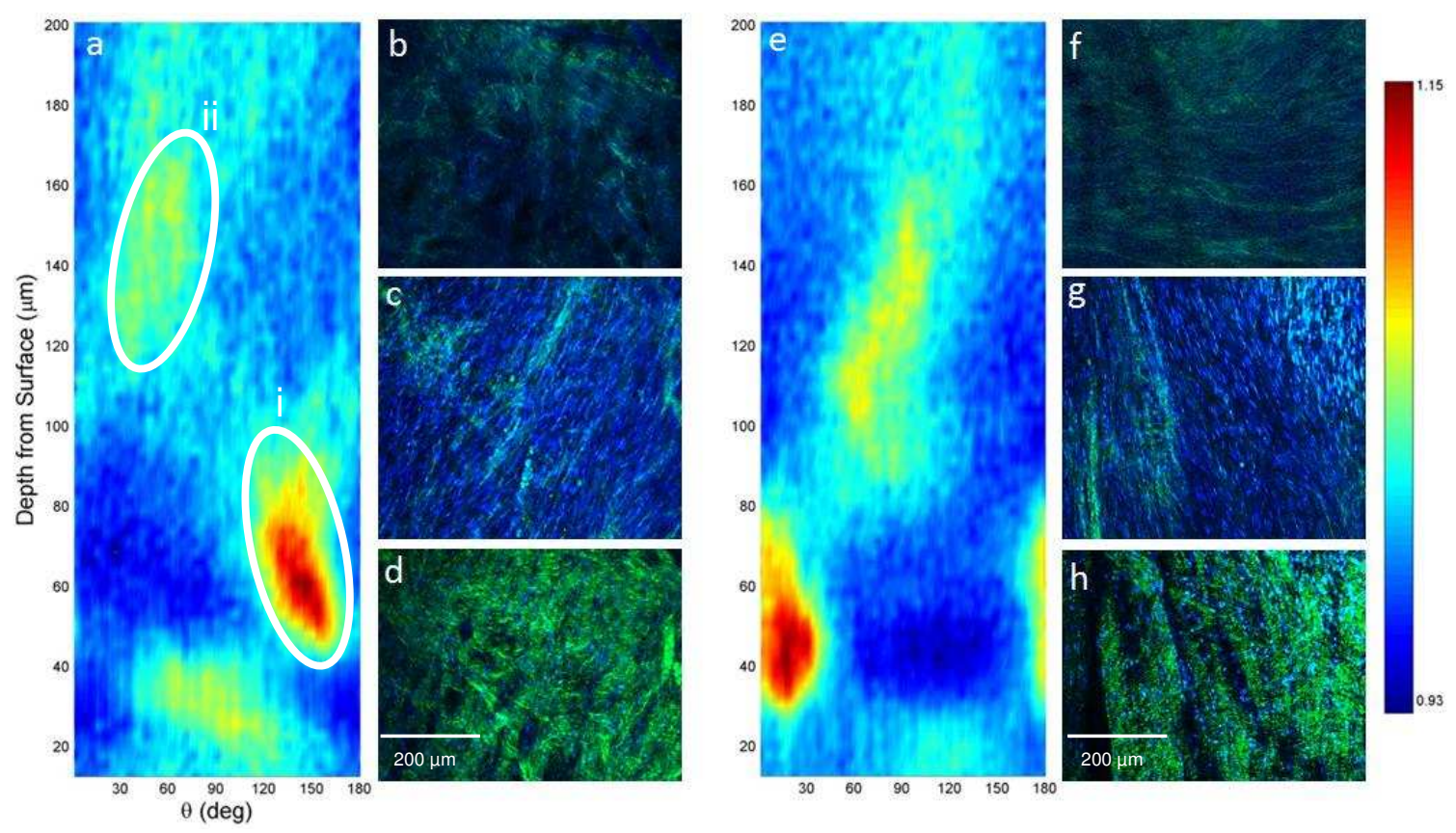

Figure 5$$
\text { . }
$$ 
a

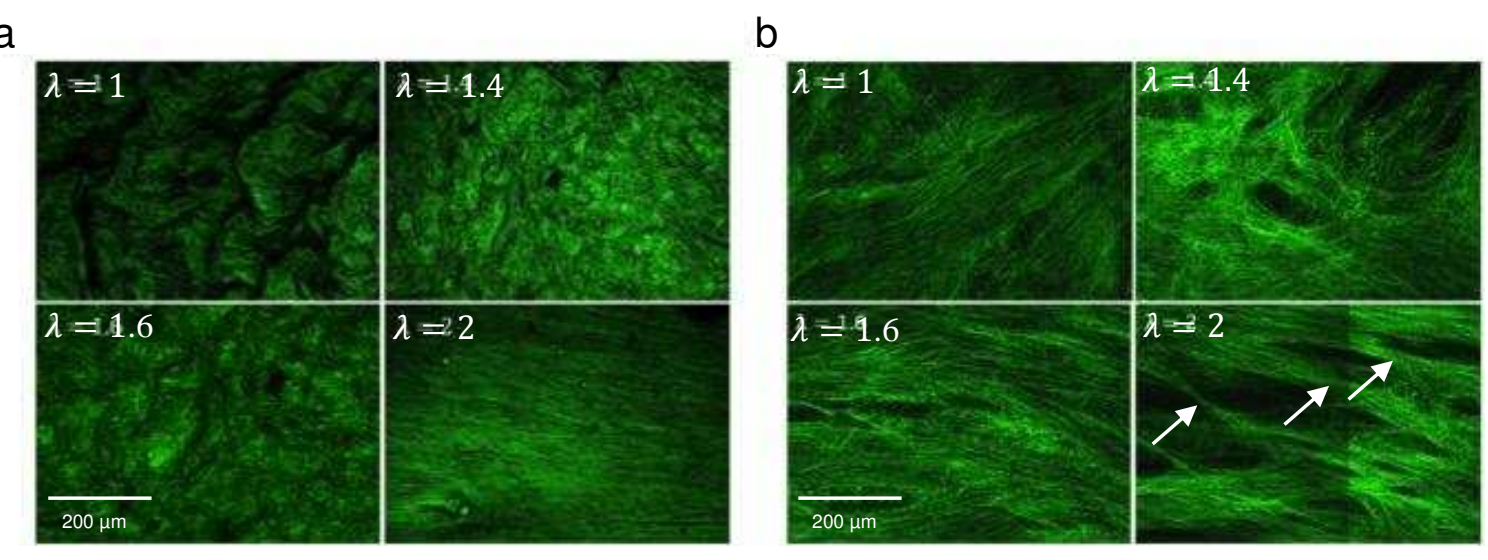

b

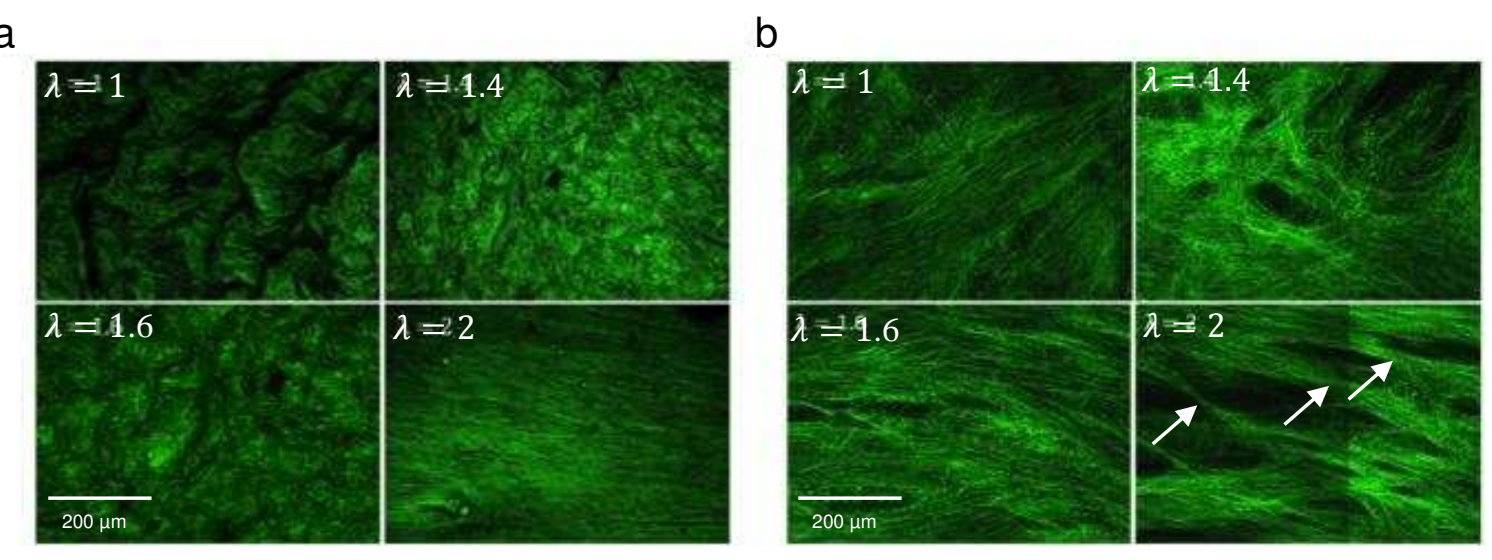

Figure 6

$a$ 


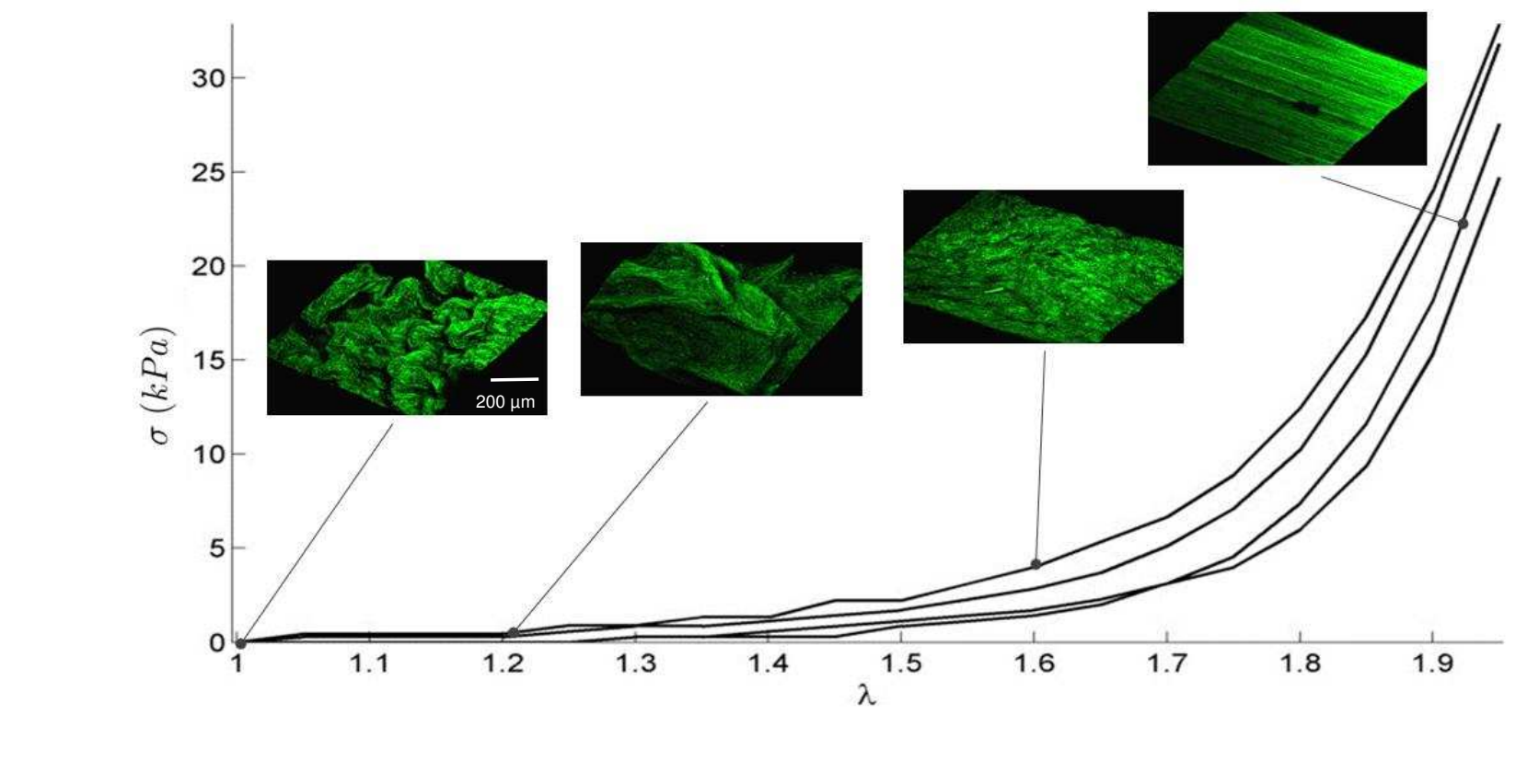

Figure 7

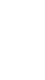

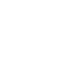

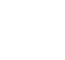

(1)

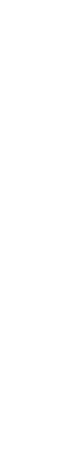
(1) ( 
Figure 8

Resting State

Lamina Propria

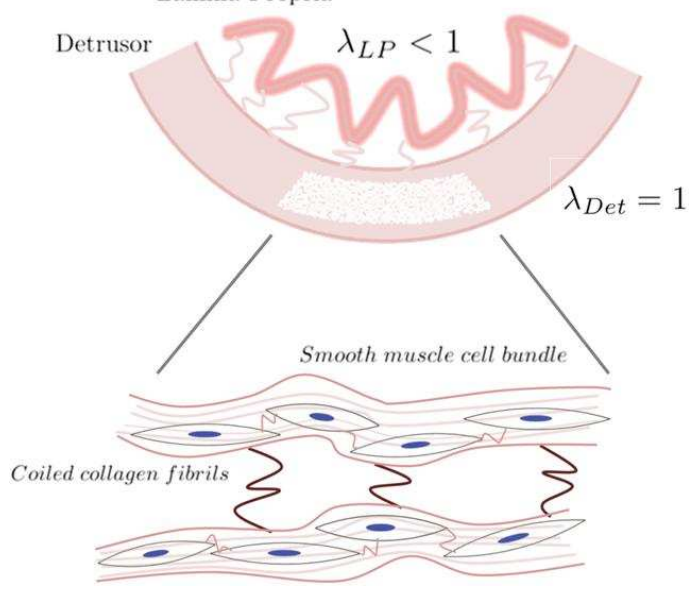

Distended State

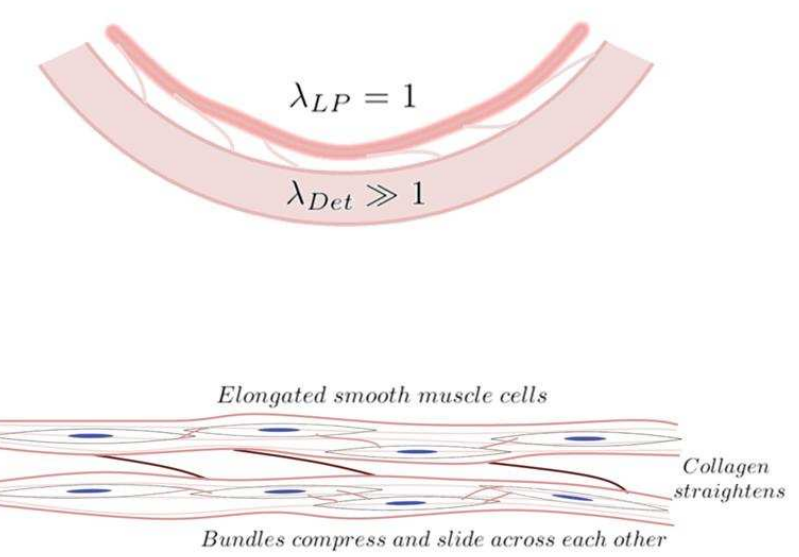




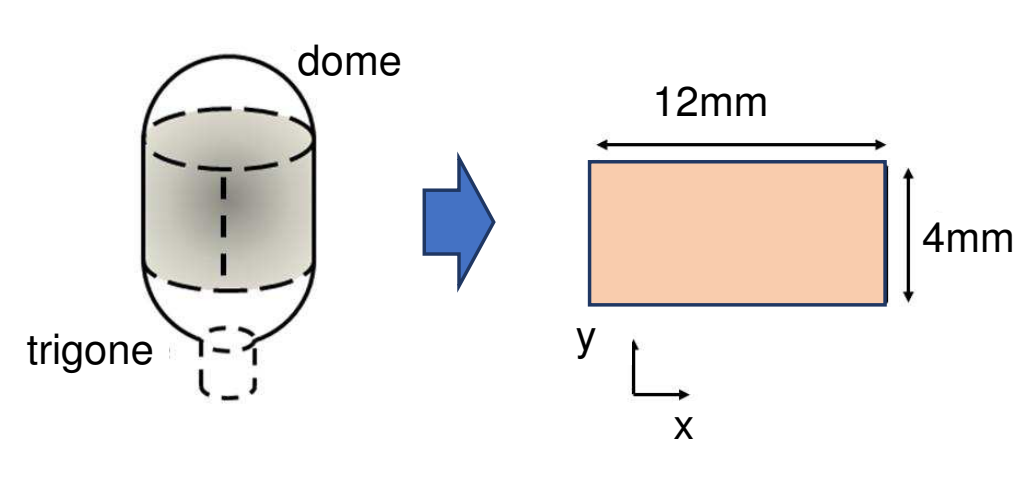

Murine bladders

5

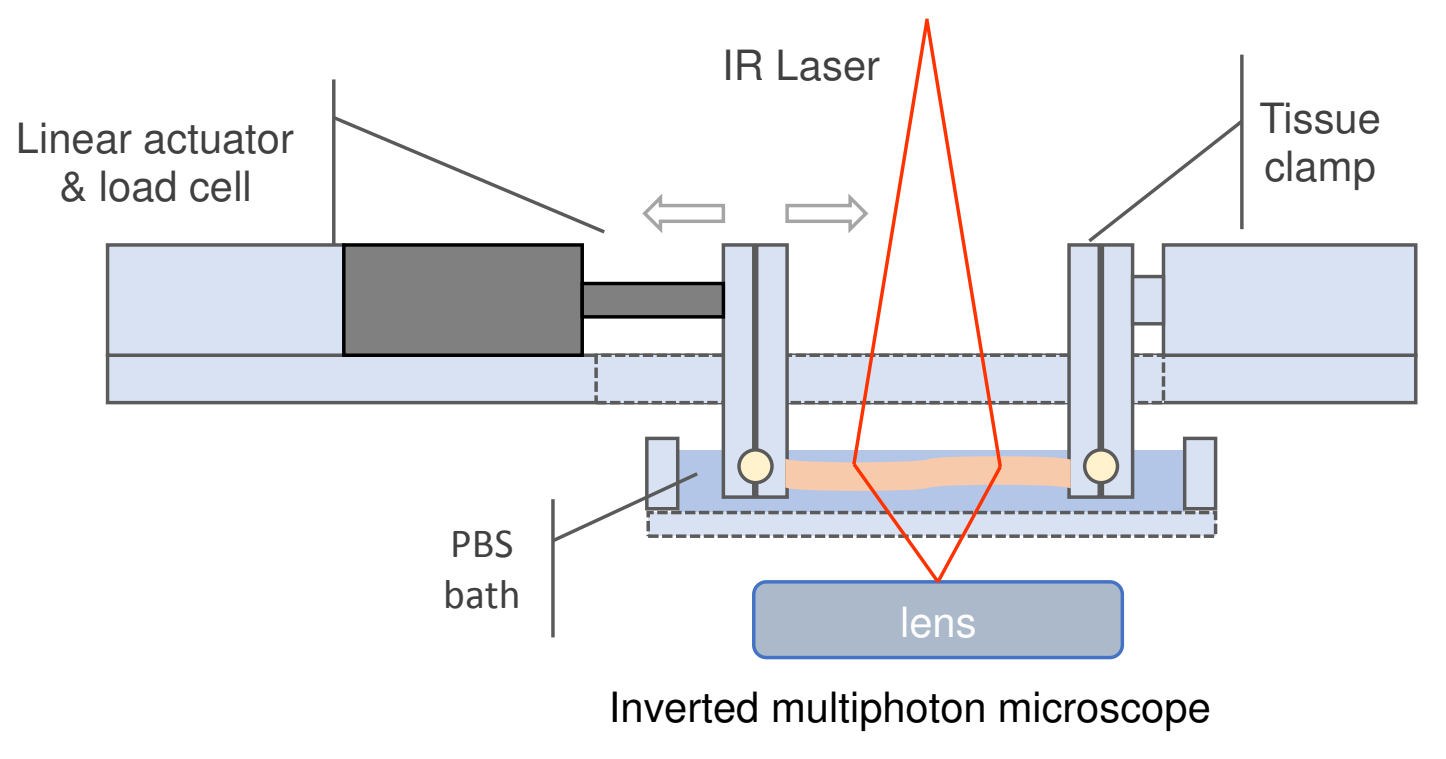

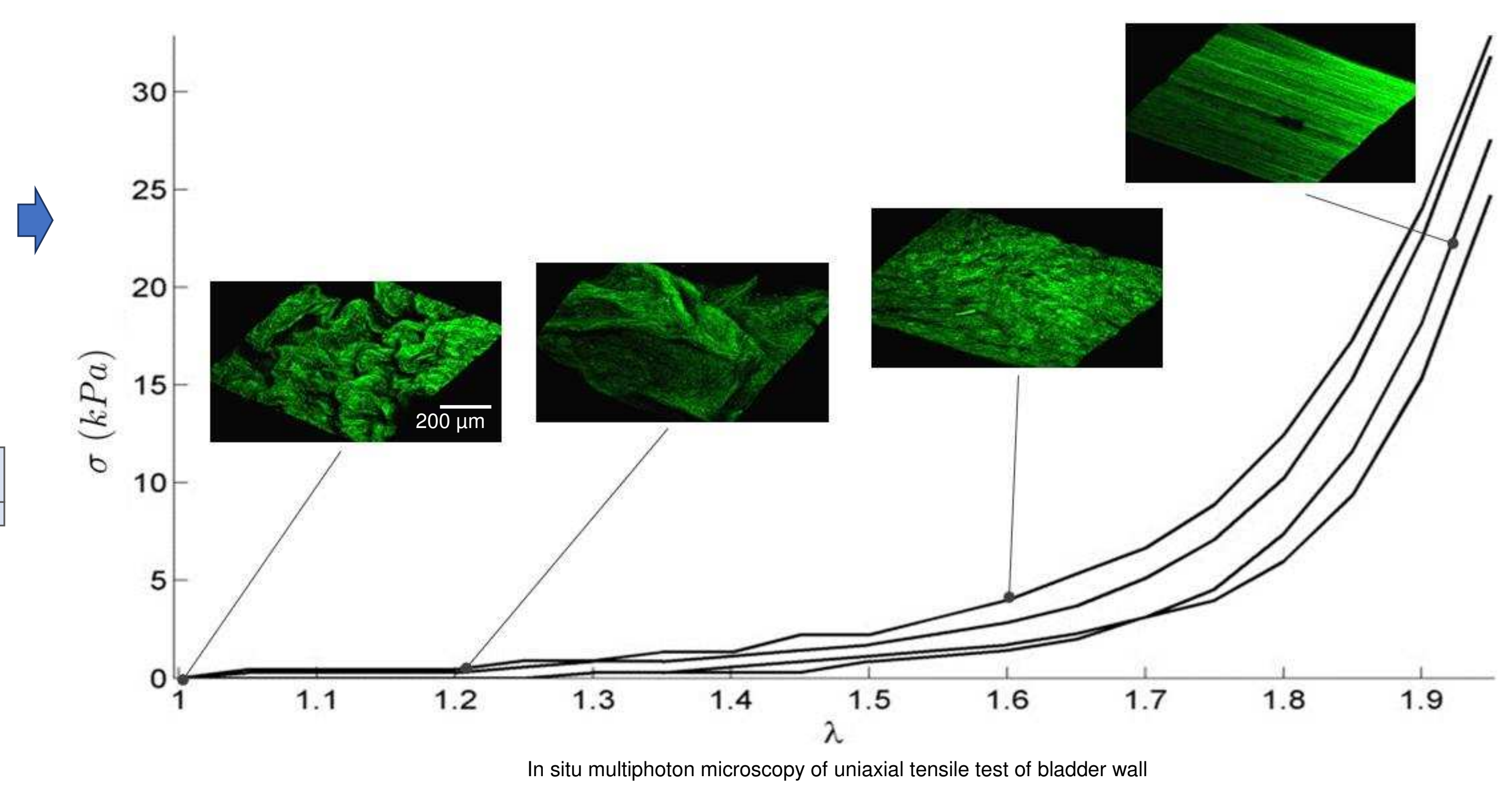

